\title{
Current Status and Advances in Imaging Evaluation of Neoadjuvant Chemotherapy of Breast Cancer
}

\author{
Yixin Hu, MD ${ }^{a}$, Fei Li, MD ${ }^{a}$, Jianhua Zhou, MD ${ }^{a, *}$ \\ ${ }^{a}$ Department of Ultrasound, Sun Yat-Sen University Cancer Center, State Key Laboratory of Oncology in South China, Collaborative \\ Innovation Center for Cancer Medicine, Guangzhou, China
}

Received November 06, 2019; revision received January 14, 2020; accepted February 02, 2020.

\begin{abstract}
It is particularly important to evaluate the efficacy of neoadjuvant chemotherapy (NAC) for breast cancer. This article reviews the current status and progress of imaging evaluations regarding the efficacy of NAC in women with breast cancer, including mammography, ultrasound (US), computed tomography (CT), magnetic resonance imaging (MRI), positron emission tomography (PET-CT) and radiomics. Each imaging method has value for evaluating the efficacy of NAC for breast cancer, but certain defects and limitations. Therefore, the optimal selection will employ a combination of multiple imaging methods that will not only benefit patients but also avoid the unnecessary waste of medical resources.
\end{abstract}

Key words: Neoadjuvant therapy; Breast neoplasms; Mammography; Ultrasonography; Computed tomography; MRI; PET-CT

Advanced Ultrasound in Diagnosis and Therapy 2021;01:018-025

DOI: $10.37015 / A U D T .2021 .190036$

$\mathrm{B}$ reast cancer is one of the most common malignant tumors among women, and its incidence is increasing annually. For example, the incidence rate of breast cancer increased by $0.3 \%$ per year in the US over the last 5 years (2012-2016), largely because of rising rates of local stage and hormone receptor-positive disease. Therefore, it is of great importance to find efficient and precise treatments of local-stage breast cancer with different molecular subtypes $[1,2]$. Neoadjuvant chemotherapy (NAC) for breast cancer is primarily used to treat locally advanced breast cancer before surgery and is indicated for patients with breast cancer in clinical stages II and III. NAC not only downstages the tumor, which helps to increase the choice of surgical methods, but also tests the response of chemotherapy in advance, while serving as an important research tool to screen for efficacy predictive molecules and pharmacokinetic parameters. In addition, it can directly verify the drug efficacy through repeated biopsy [3]. A recent meta-analysis conducted by the US Food and Drug Administration reviewed work that included 12 international centers and enrolled 11,955 patients.
The results confirmed that the survival rate of those who achieved complete pathological remission after receiving NAC was significantly improved [4]. Therefore, evaluating the efficacy of NAC is particularly important. Although pathology is the gold standard evaluation, it is invasive, slow, and difficult to perform during chemotherapy $[5,6]$. Clinical palpation is simple, but it is highly subjective and relies solely on palpable size of the mass. As a noninvasive and reproducible examination method, imaging has value when evaluating the efficacy of NAC. Numerous studies have reported that different imaging methods, including mammography, ultrasound (US), computed tomography (CT), nuclear magnetic resonance imaging (MRI), positron emission tomography (PET-CT) and radiomics, have beneficial clinical value for the evaluation of NAC efficacy. However, no standard examination method is recognized for the efficacy evaluation of NAC in clinical practice. This article reviews the current status and progress of imaging evaluations regarding the efficacy of NAC for women with breast cancer.

\footnotetext{
${ }^{*}$ Corresponding author: Department of Ultrasound, Sun Yat-Sen University Cancer Center, State Key Laboratory of Oncology in South China, Collaborative Innovation Center for Cancer Medicine, 651 Dongfeng Road East, Guangzhou, 510060, China.

e-mail: zhoujh@sysucc.org.cn unrestricted use, distribution and reproduction in any medium provided that the original work is properly attributed.
} 


\section{Mammography}

Mammography has been widely used in clinical practice given its advantages of simple operation, and affordability. It clearly shows the microcalcifications of breast cancer [7] and can be used to evaluate NAC efficacy by comparing the changes in breast mass characteristics, such as size, shape, and calcification (Fig. 1) as well as the changes in perilesional and axillary lymph nodes before and after NAC. Chagpar et al. [8] retrospectively analyzed the data of 189 patients and found that mammography was significantly superior to clinical palpation; however, its measured post-NAC residual lesion size was only moderately correlated with the pathologically measured size after surgery (correlation coefficient $r=0.41$ ), and the diagnostic accuracy was only $70 \%$. Liu et al. [9] reported that the change rate of mass was $89.3 \%$; of these changes, $87.5 \%$ of round masses showed irregular changes, the edges of $93.7 \%$ of fuzzy masses became clear, the burr of $90.5 \%$ of masses with peripheral burr became shorter or even disappear, and $74.2 \%$ of lesion calcifications changed in range, number, and distribution. Thus, mammography was expected to be used to evaluate the efficacy of NAC. However, mammographic assessment on the efficacy of NAC has limitations. For example, its accuracy is dependent on whether the mass boundary is clearly visualized before treatment. Huber et al. [10] found that the accuracy of assessing the rate of masses changing after treatment was highly correlated to masses with clear boundaries before treatment $(r=0.77)$, whereas the correlation was low for masses with unclear boundaries $(r=-0.19)$. In addition, lesion calcifications can increase, decrease, or remain unchanged after NAC [11], making it difficult to evaluate efficacy. Moreover, mammography irradiates the human body, it is not accurate enough to detect multicentric breast cancer, and it does not differentiate well between tumor residue after chemotherapy and chemotherapyinduced fibrosis [12]. Therefore, its application value for evaluating the efficacy of NAC in breast cancer is worth exploration but requires further refinement.

\section{Ultrasound}

\section{Routine ultrasonography}

Routine ultrasonography is primarily used to observe changes in the location, number, size, morphology, boundary, internal echo, and posterior echo of breast mass. Keune et al. [13] studied 192 patients with breast cancer treated with NAC and found that ultrasound detected $91.3 \%$ of all cases with tumor remnants after chemotherapy, whereas X-ray only detected $51.9 \%$ of all cases. Thus, the authors argued that ultrasound was more accurate than X-ray while measuring the size of the residual tumor after chemotherapy (Fig. 1). Roubidoux MA [14] used ultrasound to measure the maximum diameter of the mass before and after NAC to calculate the reduction rate $(\%)$ of tumor diameter and compared that value with the histopathological efficacy of NAC. The author found that ultrasound had a higher sensitivity while evaluating the efficacy of NAC for breast cancer by measuring the change in the size of the mass and reflected the efficacy of NAC more accurately; moreover, other studies [15] have supported this view. Many reports have previously documented the assessment of NAC efficacy via color Doppler by observing the changes in blood flow signal, maximum blood flow velocity (Vmax), and the resistance index (RI); however, recent studies have shown that the accuracy of its measurement is low, and the application value is not high. However, the ability of conventional ultrasound to predict pathological complete remission after NAC does not significantly
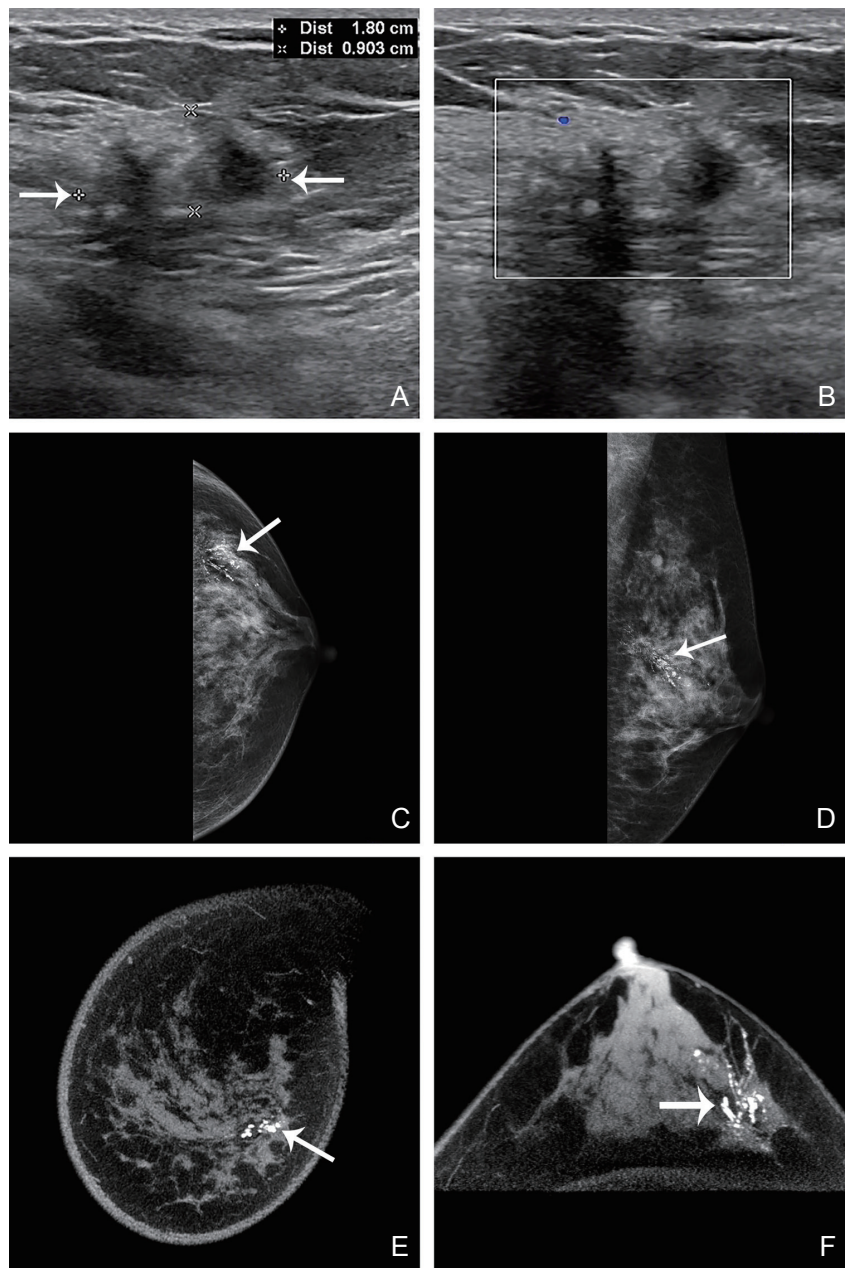

Figure 1 A 29-year-old women with a left breast lesion which was confirmed as invasive breast cancer. After completion of neoadjuvant chemotherapy, Transverse ultrasound (A and B) showed a heterogeneous hypoechoic lesion (arrows) without blood flow signal, likely reflecting residual tumor. Mammography $(\mathrm{C}$ and $\mathrm{D})$ and $\mathrm{CT}$ examination $(\mathrm{E}$ and $\mathrm{F}$ ) showed microcalcifications (arrows) correlating with ultrasound findings. Pathological examination after surgery confirmed residual tumor. 
differ from that of X-ray, and the accuracy of both is approximately $80 \%$ [13]. Studies have shown that the predictive power of the combined application of the two is better than that of any single measurement [16]. Although conventional ultrasound has some value when evaluating the efficacy of NAC, its demonstration of calcification is not as effective as mammography, and it cannot differentiate residual tumors after chemotherapy from chemotherapy-induced fibrosis [17].

\section{Contrast-enhanced ultrasound (CEUS)}

CEUS is able to demonstrate the distribution process of contrast agents in tumor blood vessels (especially tumor microvessels) in real time and dynamically through the intravenous injection of ultrasound contrast agents that can reflect the distribution of, survival of, and changes to tumor microvessels $[18,19]$. Numerous recent publications have reported that CEUS qualitatively and quantitatively assesses the changes in tumor blood perfusion after NAC in patients with breast cancer. After NAC, the use of CEUS imaging to measure the size of residual tumor is more accurate than conventional ultrasound, especially in areas where the center of the tumor has undergone apoptosis without liquefaction necrosis. Furthermore, CEUS better shows areas without enhancement that cannot be identified using conventional ultrasound [20-22]. The sensitivity, specificity, positive predictive value, and negative predictive value of CEUS in predicting the complete remission of cases following NAC have reached as high as $80 \%, 98 \%, 88.9 \%$, and $96 \%$, respectively [23]. Compared with two-dimensional CEUS, three-dimensional CEUS (3D-CEUS) more accurately reflects the changes in the internal perfusion of the tumor and tumor blood vessels before and after NAC in women with breast cancer. Chen Man [24] used 3D-CEUS to evaluate the efficacy of NAC in women with breast cancer. Compared with pre-NAC, the 3D-CEUS score after NAC was significantly decreased. The sensitivity of using the 3D-CEUS score to evaluate the efficacy of NAC in women with breast cancer was $100.0 \%$. However, this method is expensive, has currently not been validated in appropriately sized multicenter trials, and has not been widely used in the clinical evaluation of NAC efficacy.

\section{Ultrasound elastography}

Ultrasound elastography can further evaluate the degree of softness/hardness of objects based on conventional ultrasound that includes the following technologies (Fig. 2). (1) Acoustic radiation force impulse (ARFI) imaging technology is used to evaluate NAC efficacy with regard to breast cancer by measuring the softness/hardness of the internal and marginal tissues of tumors, including the virtual touch tissue image (VTI) technique and the virtual touch tissue quantification (VTQ) technique. (2) Real-time shear wave elastography is used to reflect NAC efficacy with regard to breast cancer by measuring the absolute value of Young's modulus, a value that reflects tissue elasticity. Greater values of Young's modulus denote higher elastic coefficients (i.e., harder objects). Hayashi [25] found that breast cancers with low elastographic measurement values (i.e., softer tumors) had significantly higher pathological complete remission rates after NAC than those with high measurement values (i.e., harder tumors), indicating that elastography has value for NAC efficacy prediction. Evans et al. [26] found a significant correlation between the shear wave elastography measurement values before NAC treatment and the complete remission rate of cases after treatment in cases of non-progressive breast cancer. Han et al. [27] found that the elastographic measurement values of tumors with effective treatment were significantly reduced after 2 courses of NAC treatment, but no such difference was found in the group with ineffective treatment. However, many factors affect the accuracy of breast ultrasound elastography, such as the depth and size of the lesion and the background of the perilesional tissue, whereas the size and depth of the lesions usually change after NAC treatment, which limits its application when evaluating the efficacy of NAC.

\section{Ultrasonic spectrum analysis}

The ultrasonic spectrum analysis seeks to extract relevant tissue information using the radio-frequency (RF) echo signal to perform a fast Fourier transform and a spectrum analysis. Ultrasound RF signals include single-frame RF signals and RF time-series signals, and numerous previous studies have confirmed that RF signals contain detailed information related to the tissue microstructure. Therefore, RF signal analysis can be used to observe the changes in microstructure of tumor tissue before and after chemotherapy [28, 29]. Tran et al. [28] found that the data from a single-frame RF signal analysis showed significant difference one week after NAC in patients with breast cancer, indicating that it can be used for efficacy evaluation. Sadeghi-Naini et al. [28] found that using the changing rate of RF single-frame signal analysis data better distinguished chemotherapy responsive tumors from chemotherapy refractory tumors after four weeks of NAC treatment with a $100 \%$ sensitivity and an $83.3 \%$ specificity. Ultrasound RF time-series signals are better able to assess tissue microstructure than single-frame RF signals, but their evaluation of chemotherapy efficacy in patients with breast cancer remains in the experimental stage. Clearly, 
ultrasound RF signal analysis is an emerging technique, and its application value for NAC evaluation in patients with breast cancer must be further explore.

\section{CT scan}

At present, CT scanning has evolved into multislice spiral CT scanning. The images obtained from this method have the characteristics of wide range, high spatial resolution, and high contrast resolution; moreover, they can be used to reconstruct coronal and sagittal multiplanar images, which have certain application values for evaluating the efficacy of NAC in patients with breast cancer (Fig. 1). CT can be used with high accuracy (up to $88 \%$ ) morphological assessment, including lesion size [31]. Xu Min et al. [32] compared the range of residual lesions assessed using multi-slice spiral CT with the range confirmed by the histopathological diagnosis of breast cancer after NAC treatment and found that the consistency rate was as high as $90.9 \%$. Unenhanced CT accurately reflects the changes in breast cancer lesions and lymph nodes before and after NAC, and enhanced $\mathrm{CT}$ reflects vascularity of breast cancers before and after NAC treatment. Although CT has value while evaluating the efficacy of NAC in patients with breast cancer, it also has deficiencies. For example, CT has a lower display rate of microcalcification in breast cancer lesions than mammography does, and patients must receive more radiation during the examination, which somewhat limits its clinical application.
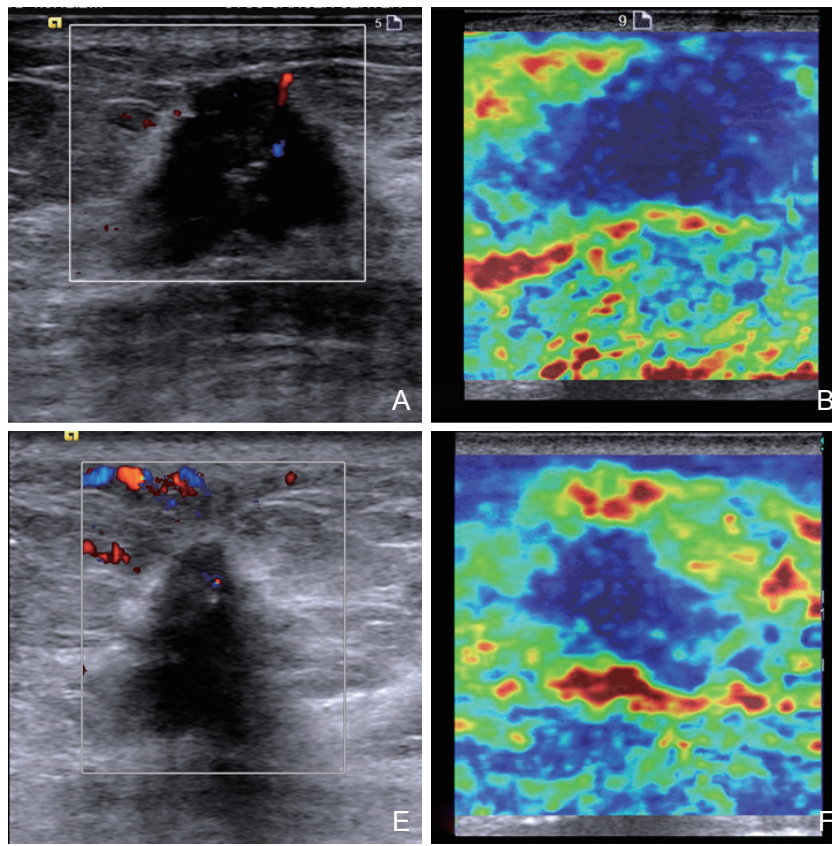
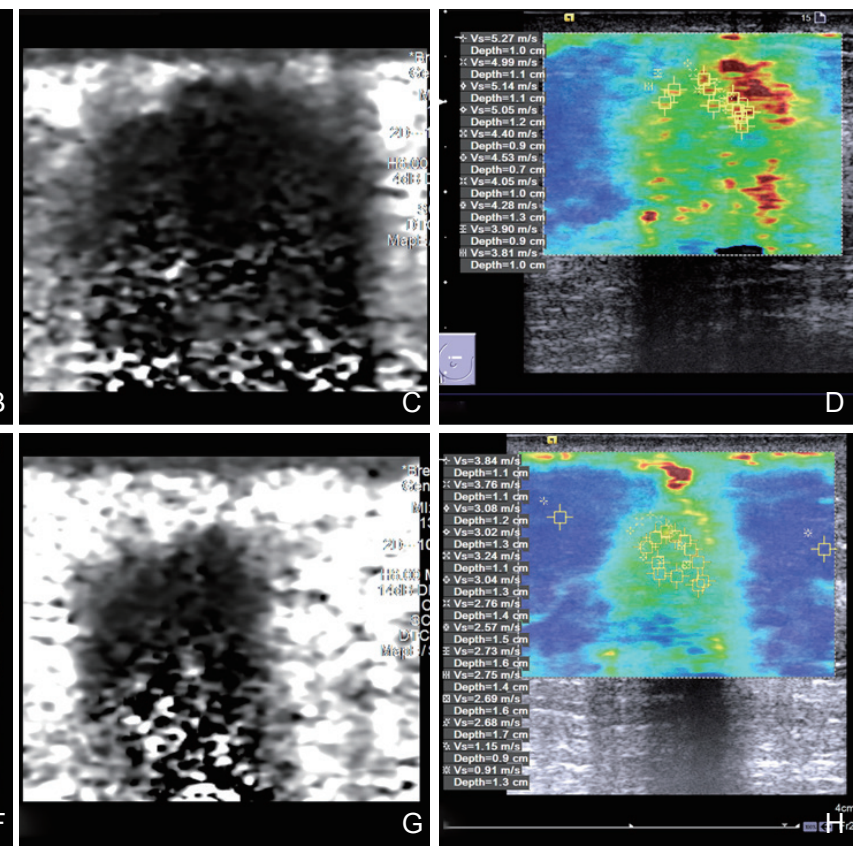

Figure 2 A 44-year-old women with a left breast lesion which was confirmed as invasive breast cancer. Before neoadjuvant chemotherapy, a solid, hypoechoic lesion with well-defined margin was detected with conventional ultrasound (A). The elasticity imaging (EI, B) and the virtual touch tissue imaging (VTI, C) reflected tissue elasticity was hard. In virtual touch tissue quantification (VTIQ), the highest shear wave velocity was 5.27 m/s (D). After two weeks of neoadjuvant chemotherapy, the lesion size decreased and the margin became ill-defined (E). The EI and VTI reflected tissue elasticity was still hard (F and G), while in VTIQ, the highest shear wave velocity decreased to $3.84 \mathrm{~m} / \mathrm{s}(\mathrm{H})$.

\section{MRI}

MRI not only accurately measures the size and morphology of tumors but also provides information on the blood supply, physiological, and biochemical metabolic information within the tumor. These methods include dynamic contrast-enhanced MRI (CE-MRI), diffusion-weighted MRI (DWI-MRI), hydrogen proton magnetic resonance spectroscopy, and magnetic resonance perfusion imaging, which can be used to more accurately evaluate the efficacy of NAC in patients with breast cancer. At present, however, more studies have focused on CE-MRI and DWI-MRI (Fig. 3).

\section{Dynamic CE-MRI}

CE-MRI can be used to detect angiogenesis during tumor progression and evaluate therapeutic efficacy by observing changes in microcirculation during tumor treatments. CE-MRI has been widely used in the literature to detect residual breast cancer following NAC. A meta-analysis that summarized 44 studies conducted between 1990 and 2008 with a total enrollment of 2,050 cases showed that CE-MRI had a higher sensitivity (sensitivity $83 \%-87 \%$ ) for the detection of residual 
lesions after NAC, although significant variation was found among studies with different specificities (specificity 54\%-83\%) [33]. Cho et al. [34] found that a voxel-based parametric response map analysis of DCEMRI better predicted treatment-responsive tumors after the first course of NAC among patients with breast cancer. CE-MRI texture analysis is a quantitative assessment method for tumor heterogeneity, and a texture analysis based on enhanced and T2-weighted sequences may have better clinical prospects regarding the evaluation of NAC to treat breast cancer. Specifically, a small sample of 35 patients with locally advanced breast cancer was conducted by $\mathrm{Wu}$ et al. [35], who found that the rapid regression of areas of significant heterogeneity in tumors predicted the effectiveness of chemotherapy. Another study that included 61 patients with breast cancer treated with NAC showed that if the tumor heterogeneity changed on T2-weighted sequences during treatment using a T2-weighted sequence-based texture analysis, then it was more closely correlated with treatment effectiveness, and this correlation was more pronounced among patients with triple-negative breast cancer [36]. However, DCE-MRI may also underestimate residual tumor after chemotherapy, possibly because chemotherapy drugs have an anti-angiogenic effect, and the decrease of blood vessels and the increase of fibrous tissue components after chemotherapy weakens tumor enhancement or changes the enhancement pattern [37].
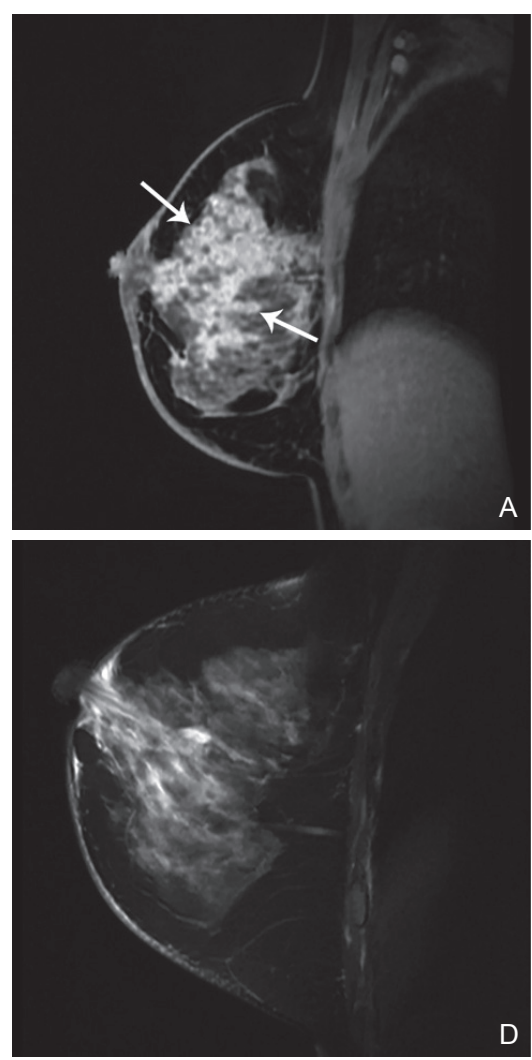
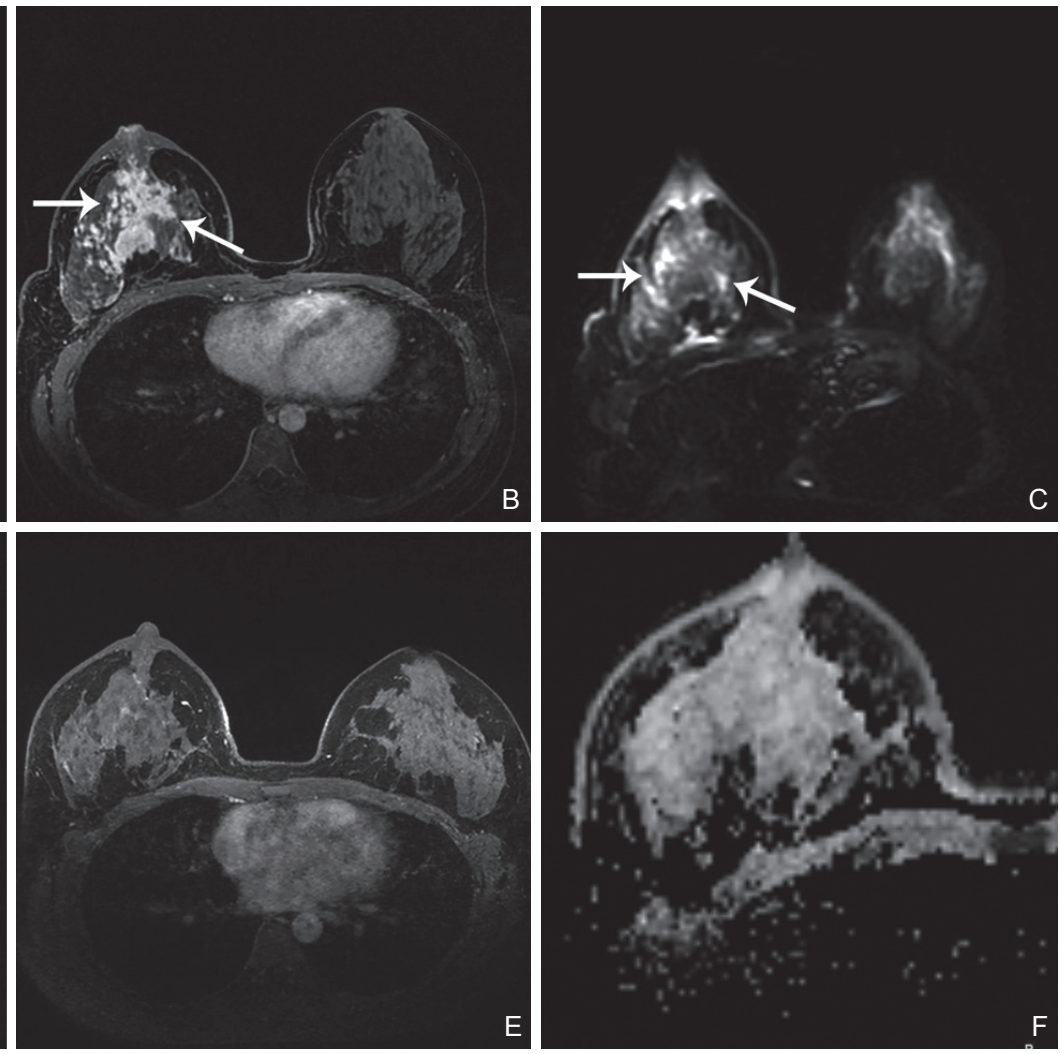

Figure 3 A 57-year-old women with multiple right breast lesions that underwent percutaneous biopsy, revealing invasive breast cancer. Before neoadjuvant chemotherapy, CE-MRI (A and B) showed area of clumped enhancement (arrows) with regional distribution, and DWI-MRI (C) showed heterogeneous masses (arrows) of right breast. After completion of neoadjuvant chemotherapy, CE-MRI (D and E) showed no residual enhancement, and DWI-MRI (F) showed no mass of right breast. Pathological examination after surgery confirmed complete pathologic response

\section{Diffusion-weighted MRI}

DWI-MRI is an imaging modality that measures the movement of water molecules. DWI-MRI images are quantitatively analyzed by determining the apparent diffusion coefficient (ADC) of water molecules within tissue. After effective chemotherapy, tumor cells die, resulting in a decreased cell density and increasing extracellular space. Thus, ADC values increase with effective NAC [38]. Based on these principles, DWI-
MRI can be used for the early assessment or prediction of NAC efficacy in patients with breast cancer. One study of 53 patients with breast cancer showed that after receiving $\mathrm{NAC}$, the $\mathrm{ADC}$ value of the chemotherapyresponsive group was significantly lower than that of the chemotherapy-refractory group [39]. Another metaanalysis that summarized the literature documenting 34 studies indicated that sensitivities of DWI-MRI and CEMRI were $93 \%$ and $68 \%$ respectively, and specificities 
were $82 \%$ and $91 \%$ respectively, suggesting that the diagnostic efficacy of DWI-MRI was partially better than that of CE-MRI [40]. However, DWI-MRI also has limitations. At present, the greatest controversy in this area is the selection of the region of interest (ROI) for measurement. The position, size, and depth of the ROI significantly affect the accuracy and repeatability of the ADC measurement values. In addition, because the detection of tumor response to chemotherapeutic agents requires dynamic tracking and repeated examinations, which may be difficult due to the cost of MRI examination and longer examination time. Furthermore, contrast agents that can cause allergic reactions must be injected during the examination. Thus, these deficiencies have somewhat limited its widespread adoption in clinical practice.

\section{Positron emission tomography (PET-CT)}

18 F-FDG PET imaging is a functional imaging technique that can be used for the study and detection of the biological changes in tumors under physiological conditions that do not affect the homeostasis of the internal environment of the human body. Tumors that respond to chemotherapy undergo significant changes in their metabolism before their volume is altered. As such, PET-CT has value when evaluating the NAC efficacy with regard to breast cancer. PET$\mathrm{CT}$ has been widely used for the clinical diagnosis and treatment of breast cancer [41]. PET-CT can be used to track tumor treatment response as well as to timely or even quantitatively evaluate tumor metabolism, proliferation, drug resistance, and receptor status with which we can observe the early subclinical response of tumors to treatment and evaluate the short- and longterm efficacies after treatment to provide a basis for developing individual chemotherapy regimens and assessing prognosis. One study of 40 patients with invasive ductal carcinoma showed that PET-CT predicted the final chemotherapy efficacy because the relative changing rate of the standardized uptake value (SUV) of the tumors, which eventually achieved pathological complete remission after patients underwent two courses of NAC, was significantly higher than that of the tumors that eventually failed to achieve pathological complete remission [42]. A meta-analysis that summarized 19 papers including a total of 920 patients indicated that the diagnostic efficacy of PET-CT for the early evaluation of chemotherapy efficacy in women with breast cancer was not high; moreover, its sensitivity, specificity, positive predictive value, and negative predictive value were $84 \%, 66 \%, 50 \%$, and $91 \%$, respectively [43]. The biggest factor that contributed to these results was the low spatial resolution of PET-CT for superficial masses. The literature reports that the sensitivity, specificity, and accuracy of PET-CT regarding the diagnosis of breast cancer lesions with diameters ranging from 3.1 to 8.0 $\mathrm{mm}$ are $93.3 \%, 90.9 \%$, and $100 \%$, respectively [43]. However, its diagnostic efficacy for masses less than $1 \mathrm{~cm}$ is significantly reduced and shows a high false negative rate. In addition, the cost of PET-CT is high, the radioactive contrast agent must be injected, and the examination process is also radioactive, which all limit its repeated use during the efficacy evaluation.

\section{Radiomics}

Radiomics is a newly-evolved field of medical imaging analysis, meaning conversions of digital medical images into mineable high-dimensional data, including both semantic and agnostic features. As is known, solid tumors are highly heterogeneous at the phenotypic, physiologic, and genomic levels, which is regarded as the main reason for resistance to tumor treatment. Radiomics enables quantitative measurement of intratumoral and intertumoral heterogeneity and reveals the relationships between information in biomedical images and the underlying pathophysiology; and the subsequent mining of these data helps to develop models for diagnosis, prediction, or prognosis [45, 46]. Radiomics has made progresses on prediction of NAC efficacy. A retrospective study with 60 patients for training and 186 patients for validation, in which each tumor was divided into multiple spatially segregated and phenotypically consistent sub-regions on the basis of perfusion MR imaging parameters, showed that perfusion MRI radiomics predicted the recurrence-free survival (RFS) after NAC because aggressive tumors were associated with a larger volume of the poorly perfused sub-region [47]. Besides analysis of the heterogeneity within tumors, radiomics can also provide us with that of regions around tumors. Braman et al. [48] conducted radiomic textural analysis of intratumoral and peritumoral regions on pretreatment DCE-MRI and found that radiomic features strongly predicted pathological complete response (pCR) after NAC independent of choice of classifier. Equipped with artificial intelligence, radiomics makes prediction easier. Drukker et al. [49] reported that the automaticallycalculated most enhancing tumor volume by MRI radiomics could predict RFS in NAC of breast cancer at early treatment, of which performance rivaled that of combining a traditional semi-manual model, functional tumor volume (FTV), and knowledge of the pre-surgical residual cancer burden. Moreover, combining radiomic features with genomic data, so called radiogenomics, may increase diagnostic, prognostic, and predictive power. Ha et al. [50] divided 73 patients with locally 
advanced breast cancer into different clusters according to metabolic radiomics patterns and found distinctive characteristics among the clusters with regard to Ki67 expression, response to NAC, and risk of recurrence. In all, radiomics analysis epitomize the pursuit of precision medicine, which helps to provide personalized treatments with integration of quantitative information with clinical, histological, and genomic data. However, the power of radiomics depends on the size and quality of the database, requests standard acquisition and big data for reproducibility, while also risking overfitting. In the future efforts to establish standard, sharing data as well as avoiding redundant feature extractions are needed.

\section{Conclusions}

In summary, the early, correct, and objective evaluation of breast cancer response to NAC provides better options for the clinical selection of chemotherapy drugs to develop appropriate treatment regimen, which helps to reduce unnecessary drug use and decrease the toxic side effects of chemotherapy and improve the survival and quality of life of patients. Various imaging methods have application value but certain defects and limitations. Therefore, clinicians should choose appropriate imaging methods to evaluate and predict the efficacy of NAC based on actual patient conditions in clinical practice. The optimal selection as well as reasonable application and combination of multiple imaging methods will not only benefit patients but also avoid the unnecessary waste of medical resources.

\section{Conflicts of Interest}

The authors declare no conflict of interests.

\section{References}

[1] DeSantis CE, Ma J, Gaudet MM, Newman LA, Miller KD, Goding Sauer A, et al. Breast cancer statistics, 2019. CA Cancer J Clin 2019; 69: 438-51.

[2] P Wang, JF Li, WS Yue, WY Li, Luo Y. The correlation between traditional ultrasound features and the expression of estrogen receptor, progesterone receptor, human epidermal growth factor receptor-2, and Ki-67 in breast carcinoma. Advanced Ultrasound in Diagnosis and Therapy 2018; 2: 173-77.

[3] Buchholz TA, Lehman CD, Harris JR, Pockaj BA, Khouri N, Hylton NF, et al. Statement of the science concerning locoregional treatments after preoperative chemotherapy for breast cancer: a National Cancer Institute conference. J Clin Oncol 2008; 26: 791-7.

[4] Cortazar P, Zhang L, Untch M, Mehta K, Costantino JP, Wolmark N, et al. Pathological complete response and long-term clinical benefit in breast cancer: the CTNeoBC pooled analysis. Lancet 2014; 384: 164-72.

[5] Fisher ER, Wang J, Bryant J, Fisher B, Mamounas E, Wolmark N. Pathobiology of preoperative chemotherapy: findings from the National Surgical Adjuvant Breast and Bowel (NSABP) protocol
B-18. Cancer 2002; 95: 681-95.

[6] Hafiz F, Choudhury T, Kamal M, Banu SG. The spectrum of pathological changes in breast cancer following neoadjuvant chemotherapy. Mymensingh Med J 2014; 23: 272-80.

[7] Fiorica JV. Breast cancer screening, mammography, and other modalities. Clin Obstet Gynecol 2016; 59: 688-709.

[8] Chagpar AB, Middleton LP, Sahin AA, Dempsey P, Buzdar AU, Mirza AN, et al. Accuracy of physical examination, ultrasonography, and mammography in predicting residual pathologic tumor size in patients treated with neoadjuvant chemotherapy. Ann Surg 2006; 243 : 257-64.

[9] Liu J, Li LL, Zhou SB, Wang B, Hu WY, Wu XM. The implication of mammographic changes before and after neo-adjuvant chemotherapy in breast cancer. Journal of Medical Imaging, 2006, 16(6): 572-574. doi:10.3969/j.issn.1006-9011.2006.06.010. [In Chinese]

[10] Huber S, Wagner M, Zuna I, Medl M, Czembirek H, Delorme S. Locally advanced breast carcinoma: evaluation of mammography in the prediction of residual disease after induction chemotherapy. Anticancer Res 2000; 20: 553-8.

[11] Kim YS, Chang JM, Moon HG, Lee J, Shin SU, Moon WK. Residual mammographic microcalcifications and enhancing lesions on MRI after neoadjuvant systemic chemotherapy for locally advanced breast cancer: correlation with histopathologic residual tumor size. Ann Surg Oncol 2016; 23: 1135-42.

[12] Shah SK, Shah SK, Greatrex KV. Current role of magnetic resonance imaging in breast imaging: a primer for the primary care physician. $J$ Am Board Fam Pract 2005; 18: 478-90.

[13] Keune JD, Jeffe DB, Schootman M, Hoffman A, Gillanders WE, Aft RL. Accuracy of ultrasonography and mammography in predicting pathologic response after neoadjuvant chemotherapy for breast cancer. Am J Surg 2010; 199: 477-84.

[14] Roubidoux MA, LeCarpentier GL, Fowlkes JB, Bartz B, Pai D, Gordon SP, et al. Sonographic evaluation of early-stage breast cancers that undergo neoadjuvant chemotherapy. $J$ Ultrasound Med 2005; 24: 885-95.

[15] Singh G, Kumar P, Parshad R, Seith A, Thulkar S, Hosten N. Role of color Doppler indices in predicting disease-free survival of breast cancer patients during neoadjuvant chemotherapy. Eur J Radiol 2010; 75: e158-62.

[16] Peintinger F, Kuerer HM, Anderson K, Boughey JC, MericBernstam F, Singletary SE, et al. Accuracy of the combination of mammography and sonography in predicting tumor response in breast cancer patients after neoadjuvant chemotherapy. Ann Surg Oncol 2006; 13: 1443-9.

[17] Londero V, Bazzocchi M, Del Frate C, Puglisi F, Di Loreto C, Francescutti G, et al. Locally advanced breast cancer: comparison of mammography, sonography and MR imaging in evaluation of residual disease in women receiving neoadjuvant chemotherapy. Eur Radiol 2004; 14: 1371-9.

[18] Rim A, Chellman-Jeffers M. Trends in breast cancer screening and diagnosis. Cleve Clin J Med 2008; 75 Suppl 1: S2-9.

[19] XM Chen, JY Zhong, ZM Hu, W Wei, WH Yin, LG Cui, et al. Translymphatic contrast-enhanced ultrasound in combination with blue dye injection is feasible for detection and biopsy of sentinel lymph nodes in breast cancer. Advanced Ultrasound in Diagnosis and Therapy 2018; 2: 94-100.

[20] Amioka A, Masumoto N, Gouda N, Kajitani K, Shigematsu H, Emi A, et al. Ability of contrast-enhanced ultrasonography to determine clinical responses of breast cancer to neoadjuvant chemotherapy. Jpn $J$ Clin Oncol 2016; 46: 303-9.

[21] Cao X, Xue J, Zhao B. Potential application value of contrastenhanced ultrasound in neoadjuvant chemotherapy of breast cancer. 
Ultrasound Med Biol 2012; 38: 2065-71.

[22] Corcioni B, Santilli L, Quercia S, Zamagni C, Santini D, Taffurelli M, et al. Contrast-enhanced US and MRI for assessing the response of breast cancer to neoadjuvant chemotherapy(). J Ultrasound 2008; 11: 143-50.

[23] Fujisawa T, Hirakata T, Yanagita Y, Iijima M, Horikoshi H, Takeuchi $\mathrm{K}$, et al. The detection of pCR after PST by contrast-enhanced ultrasonography for breast cancer. Breast Cancer 2013; 20: 75-82.

[24] Chen M, Wang WP, Jia WR, Tang L, Wang Y, Zhan WW, et al. Three-dimensional contrast-enhanced sonography in the assessment of breast tumor angiogenesis: correlation with microvessel density and vascular endothelial growth factor expression. $J$ Ultrasound Med 2014; 33: 835-46.

[25] Hayashi M, Yamamoto Y, Ibusuki M, Fujiwara S, Yamamoto S, Tomita S, et al. Evaluation of tumor stiffness by elastography is predictive for pathologic complete response to neoadjuvant chemotherapy in patients with breast cancer. Ann Surg Oncol 2012; 19: 3042-9.

[26] Evans A, Armstrong S, Whelehan P, Thomson K, Rauchhaus P, Purdie C, et al. Can shear-wave elastography predict response to neoadjuvant chemotherapy in women with invasive breast cancer? $\mathrm{Br}$ J Cancer 2013; 109: 2798-802.

[27] Jing H, Cheng W, Li ZY, Ying L, Wang QC, Wu T, et al. Early evaluation of relative changes in tumor stiffness by shear wave elastography predicts the response to neoadjuvant chemotherapy in patients with breast cancer. J Ultrasound Med 2016; 35: 1619-27.

[28] Tran WT, Childs C, Chin L, Slodkowska E, Sannachi L, Tadayyon $\mathrm{H}$, et al. Multiparametric monitoring of chemotherapy treatment response in locally advanced breast cancer using quantitative ultrasound and diffuse optical spectroscopy. Oncotarget 2016; 7 : 19762-80.

[29] Tadayyon H, Sannachi L, Gangeh M, Sadeghi-Naini A, Tran W, Trudeau ME, et al. Quantitative ultrasound assessment of breast tumor response to chemotherapy using a multi-parameter approach. Oncotarget 2016; 7: 45094-111.

[30] Sadeghi-Naini A, Papanicolau N, Falou O, Zubovits J, Dent R, Verma S, et al. Quantitative ultrasound evaluation of tumor cell death response in locally advanced breast cancer patients receiving chemotherapy. Clin Cancer Res 2013; 19: 2163-74.

[31] Moschetta M, Scardapane A, Lorusso V, Rella L, Telegrafo M, Serio $\mathrm{G}$, et al. Role of multidetector computed tomography in evaluating incidentally detected breast lesions. Tumori 2015; 101: 455-60.

[32] Min XU, Jian-Song JI, Chen-Ying LU, Chen SZ. The efficacy of CT in the evaluation of the neoadjuvant chemotherapy in patients with primary breast cancer. Journal of Medical Imaging 2012,

[33] Marinovich ML, Houssami N, Macaskill P, Sardanelli F, Irwig L, Mamounas EP, et al. Meta-analysis of magnetic resonance imaging in detecting residual breast cancer after neoadjuvant therapy. $J$ Natl Cancer Inst 2013; 105: 321-33.

[34] Cho N, Im SA, Park IA, Lee KH, Li M, Han W, et al. Breast cancer: early prediction of response to neoadjuvant chemotherapy using parametric response maps for MR imaging. Radiology 2014; 272: 385-96.

[35] Wu J, Gong G, Cui Y, Li R. Intratumor partitioning and texture analysis of dynamic contrast-enhanced (DCE)-MRI identifies relevant tumor subregions to predict pathological response of breast cancer to neoadjuvant chemotherapy. J Magn Reson Imaging 2016; 44: $1107-15$.
[36] Waugh SA, Purdie CA, Evans AJ, Lerski RA, Mcghee H, Vinnicombe SJ, et al. Response to neoadjuvant chemotherapy assessed by magnetic resonance imaging (MRI) texture analysis compared with residual cancer burden (RCB) and tumor subtype in primary breast cancer. Cancer Research 2016; 76 :

[37] Bahri S, Chen JH, Mehta RS, Carpenter PM, Nie K, Kwon SY, et al. Residual breast cancer diagnosed by MRI in patients receiving neoadjuvant chemotherapy with and without bevacizumab. Ann Surg Oncol 2009; 16: 1619-28.

[38] Galons JP, Altbach MI, Paine-Murrieta GD, Taylor CW, Gillies RJ. Early increases in breast tumor xenograft water mobility in response to paclitaxel therapy detected by non-invasive diffusion magnetic resonance imaging. Neoplasia 1999; 1: 113-7.

[39] Park SH, Moon WK, Cho N, Song IC, Chang JM, Park IA, et al. Diffusion-weighted MR imaging: pretreatment prediction of response to neoadjuvant chemotherapy in patients with breast cancer. Radiology 2010; 257: 56-63.

[40] Wu LM, Hu JN, Gu HY, Hua J, Chen J, Xu JR. Can diffusionweighted MR imaging and contrast-enhanced MR imaging precisely evaluate and predict pathological response to neoadjuvant chemotherapy in patients with breast cancer? Breast Cancer Res Treat 2012; 135: 17-28.

[41] Tatsumi M, Cohade C, Mourtzikos KA, Fishman EK, Wahl RL. Initial experience with FDG-PET/CT in the evaluation of breast cancer. Eur J Nucl Med Mol Imaging 2006; 33: 254-62.

[42] Andrade WP, Lima EN, Osório CA, do Socorro Maciel M, Baiocchi $\mathrm{G}$, Bitencourt AG, et al. Can FDG-PET/CT predict early response to neoadjuvant chemotherapy in breast cancer? Eur J Surg Oncol 2013; 39: 1358-63.

[43] Wang Y, Zhang C, Liu J, Huang G. Is 18F-FDG PET accurate to predict neoadjuvant therapy response in breast cancer? A metaanalysis. Breast Cancer Res Treat 2012; 131: 357-69.

[44] Schelling M, Avril N, Nährig J, Kuhn W, Römer W, Sattler D, et al. Positron emission tomography using [(18)F]Fluorodeoxyglucose for monitoring primary chemotherapy in breast cancer. J Clin Oncol 2000; 18: 1689-95.

[45] Gillies RJ, Kinahan PE, Hricak H. Radiomics: images are more than pictures, they are data. Radiology 2016; 278: 563-77.

[46] Crivelli P, Ledda RE, Parascandolo N, Fara A, Soro D, Conti M. A new challenge for radiologists: radiomics in breast cancer. Biomed Res Int 2018; 2018: 6120703.

[47] Wu J, Cao G, Sun X, Lee J, Rubin DL, Napel S, et al. Intratumoral spatial heterogeneity at perfusion $\mathrm{mr}$ imaging predicts recurrence-free survival in locally advanced breast cancer treated with neoadjuvant chemotherapy. Radiology 2018; 288: 26-35.

[48] Braman NM, Etesami M, Prasanna P, Dubchuk C, Gilmore H, Tiwari $\mathrm{P}$, et al. Intratumoral and peritumoral radiomics for the pretreatment prediction of pathological complete response to neoadjuvant chemotherapy based on breast DCE-MRI. Breast Cancer Res 2017; 19: 57.

[49] Drukker K, Li H, Antropova N, Edwards A, Papaioannou J, Giger ML. Most-enhancing tumor volume by MRI radiomics predicts recurrence-free survival "early on" in neoadjuvant treatment of breast cancer. Cancer Imaging 2018; 18: 12.

[50] Ha S, Park S, Bang JI, Kim EK, Lee HY. Metabolic radiomics for pretreatment (18)F-FDG PET/CT to characterize locally advanced breast cancer: histopathologic characteristics, response to neoadjuvant chemotherapy, and prognosis. Sci Rep 2017; 7: 1556. 